\title{
Extending the Nonlinear-Beam-Dynamics Concept of 1D Fixed Points to 2D Fixed Lines
}

\author{
G. Franchetti ${ }^{1}$ and F. Schmidt ${ }^{2}$ \\ ${ }^{1}$ GSI Darmstadt, Planckstrasse 1, 64291 Darmstadt, Germany \\ ${ }^{2}$ CERN CH-1211, Geneva 23, Switzerland \\ (Received 30 January 2015; published 12 June 2015)
}

\begin{abstract}
The origin of nonlinear dynamics traces back to the study of the dynamics of planets with the seminal work of Poincaré at the end of the nineteenth century: Les Méthodes Nouvelles de la Mécanique Céleste, Vols. 1-3 (Gauthier Villars, Paris, 1899). In his work he introduced a methodology fruitful for investigating the dynamical properties of complex systems, which led to the so-called "Poincaré surface of section," which allows one to capture the global dynamical properties of a system, characterized by fixed points and separatrices with respect to regular and chaotic motion. For two-dimensional phase space (one degree of freedom) this approach has been extremely useful and applied to particle accelerators for controlling their beam dynamics as of the second half of the twentieth century. We describe here an extension of the concept of 1D fixed points to fixed lines in two dimensions. These structures become the fundamental entities for characterizing the nonlinear motion in the four-dimensional phase space (two degrees of freedom).
\end{abstract}

DOI: 10.1103/PhysRevLett.114.234801

PACS numbers: 41.75.-i, 29.27.Bd

For experimental physicists, accelerators are devices that provide particle beams to a detector, or that guide two beams to collide with one another to study fundamental properties of matter at a subatomic level. It is less known that particles in a circular accelerator are subject to violent and complex dynamics, which in many aspects resemble and even exceed the complexity of the dynamics of planet motion around the sun. In accelerators, magnet nonlinearities are responsible for the nonlinear dynamics in one, two, and even three degrees of freedom. The characterization of the nonlinear motion is usually expressed in terms of the stability properties and trajectory deformation. Accelerator physicists have become familiar with properties such as dynamic aperture, fixed points, island and separatrices structures as the requirement of controlling the beam dynamics. This has led to the development of nonlinear dynamics "for accelerators" [1-4]. Particle motion can either be regular or chaotic, the latter leading to particle loss. The characterization of the regular motion and the onset of chaotic motion are therefore of utmost importance for particle accelerators [1,2].

In absence of nonlinearities the horizontal linear motion is governed by a harmonic oscillator equation of motion $x^{\prime \prime}+k_{x}(s) x=0$, where $k_{x}(s)$ is the strength of the linear focusing or defocusing elements in the accelerator, resulting in the linear horizontal tune (phase advance per turn), $Q_{x}=(1 / 2 \pi) \int_{0}^{L} \beta_{x}(s)^{-1} d s$, with $\beta_{x}(s)$ the well-known horizontal $\beta$ function incorporating the focusing system

Published by the American Physical Society under the terms of the Creative Commons Attribution 3.0 License. Further distribution of this work must maintain attribution to the author(s) and the published article's title, journal citation, and DOI. $k_{x}(s)$, and $L$ the length of the circular accelerator [5]. The particle trajectories in the $2 \mathrm{D}$ phase space are lines around a closed orbit which typically is located at zero amplitude. In the Poincaré surface of section [6] (any fixed longitudinal location $s$ ) one finds ellipses around the closed orbit.

The introduction of nonlinearities disturbs this simple and elegant structure. The motion of a particle is governed by the nonlinear equation $x^{\prime \prime}+k_{x}(s) x=-1 / n !\left[k_{n}(s) x^{n}\right]$, with $k_{n}(s)$ the strength of the nonlinearity of order $n$. The topology of the orbits in the Poincare surface of section is determined by the tune $Q_{x}$, which is now defined as the averaged phase advance per turn. For $Q_{x}$ close to the resonance $(n+1) Q_{x}=$ $m$, the topology of the orbits changes and some of the Courant-Snyder ellipses are broken into higher-order closed orbits at nonzero amplitudes (fixed points) and the particle motion in its vicinity is restricted to islands around the fixed points. Last, at some distance to these fixed points a separatrices is reached that separates the island motion from the nominal motion around the closed orbit at zero amplitude. It is interesting to note that chaotic motion develops around the separatrices due to unavoidable additional resonances as originally described by Poincaré in 1899 [6].

The Poincaré surface of section method allows a visual representation of the dynamics of a particle, which is useful for describing the motion. The control of the islands and separatrices is even used for operational schemes like the slow resonance extraction [7].

The importance of the nonlinear dynamics in accelerators has recently been emphasized with the advent of new accelerator projects $[8,9]$, which have turned the focus on machines, where properties vary with time. The phenomenon of 1D resonance crossing for high-intensity beams has been explained in terms of trapping and scattering of a 
particle interacting with islands, and extensive experimental and numerical studies have characterized the case of 1D dynamics $[10,11]$.

The reduction of the dynamics to one degree of freedom allows a visual representation of the main feature of the dynamics in terms of islands and fixed points. However, the dynamics in accelerators is actually determined in two and three degrees of freedom and the resonances excited by nonlinear components in an accelerator are found for the horizontal and vertical tunes $Q_{x}, Q_{y}$, satisfying the relation $n_{x} Q_{x}+n_{y} Q_{y}=N$. A theory describing the dynamics of a particle in two dimensions was developed in the 1950s by Schoch [4]. This theory describes how the strength of magnet nonlinearities is related to the resonant behavior of a particle: for nonlinear field components distributed around the circular accelerator, the main properties of a resonance can be related to a quantity called the "driving term." The theory predicts the resonance stop band as a function of the driving term. Despite this enormous progress, it remains unexplained how resonance structures in two or more degrees of freedom actually govern the phase space. The purpose of this Letter is to shed some new light on this issue.

The discussion of the dynamics in terms of islands and fixed points is now more difficult, the Poincare surface of section is a 4D phase space and no longer easily understandable. One can try to predict the beam evolution via computer simulations to circumvent the complicated theoretical analysis. In fact, the actual development of the treatment of nonlinear dynamics in accelerators is quite mature [12], and the present level of computer power allows demanding numerical investigation. However, the increase of computer power does not help in understanding what really happens in the multidimensional phase space if we do not know what to look for in the complex dynamics unless guided by a deeper theoretical understanding of these complexities.

In the following an approach is presented on how to characterize the dynamics in the proximity of a coupled 2D resonance in a rigorous analytic way. The technical details of this approach will be discussed in an upcoming article [13].

The simplest situation of coupled nonlinear dynamics is found when considering a circular accelerator affected by a sequence of thin sextupoles. In this case, the equations of motion reads

$$
\begin{aligned}
& \frac{d^{2} x}{d s^{2}}+k_{x}(s) x=-\frac{1}{2} k_{2}(s)\left(x^{2}-y^{2}\right), \\
& \frac{d^{2} y}{d s^{2}}+k_{y}(s) y=k_{2}(s) x y .
\end{aligned}
$$

The solution of this equation can be written as

$$
\begin{aligned}
& x(s)=\sqrt{\beta_{x} a_{x}} \cos \left[\phi_{x}(s)+\varphi_{x}\right], \\
& y(s)=\sqrt{\beta_{y} a_{y}} \cos \left[\phi_{y}(s)+\varphi_{y}\right],
\end{aligned}
$$

where $\beta_{x}, \beta_{y}$ are the well-known horizontal and vertical beta functions, and the phase advances are $\phi_{x}(s)=$ $\int_{0}^{s} \beta_{x}(s)^{-1} d s, \phi_{y}(s)=\int_{0}^{s} \beta_{y}(s)^{-1} d s$. The function $k_{2}(s)$ describes the local strength of the nonlinear errors, which excite the resonance $Q_{x}+2 Q_{y}=N$, with $N$ the harmonics of the distribution of the errors.

The quantities $a_{x}, a_{y}, \varphi_{x}, \varphi_{y}$ are the constants of linear motion. However, if we consider the system described by Eq. (1), and search for solutions of the form of Eq. (2), then $a_{x}, a_{y}, \varphi_{x}, \varphi_{y}$ are forced to become time dependent. The evolution of these "new" variables is governed by a Hamiltonian composed by a large number of harmonics, each of which has frequencies obtained as a combination of the tunes $Q_{x}, Q_{y}$. This is the Hamiltonian of the nonlinear terms in Eq. (1). The situation is hopelessly complex for an analytic treatment when all these harmonics should be included into the equations of motion. However, for a machine set close to the resonance $Q_{x}+2 Q_{y}=N$, the harmonics with frequency $Q_{x}+2 Q_{y}-N$ become very slow. As a result, only two slowly varying harmonics remain while the others "average out" quickly and are ignored. This approximation is valid close to the resonance, and for reasonable small nonlinear errors. We then take the truncated Hamiltonian (slowly varying) as an approximation of the real Hamiltonian when the system is in proximity to a third order $2 \mathrm{D}$ coupled resonance. The same procedure is used in Refs. $[4,14]$. In our study we find that $N_{j}$ sextupolar errors lead to the slowly varying Hamiltonian

$$
H_{s 1}=\Lambda \sqrt{a_{x}} a_{y} \cos \left(\alpha+2 \pi \Delta_{r} \frac{s}{L}+\varphi_{x}+2 \varphi_{y}\right),
$$

with $\Delta_{r}=Q_{x}+2 Q_{y}-N$ defined as the distance of the tunes $Q_{x}, Q_{y}$ to the resonance. Here, $\Lambda$ is the driving term of the resonance, and $\alpha$ is its orientation in the complex plane. Both these factors incorporate the effect of the distributed sextupolar errors of integrated strength $K_{2 j}$ located at position $s_{j}$. The quantity $\Lambda$, and the angle $\alpha$ determine the dynamics in this approximation.

The dynamics of the variables $a_{x}, a_{y}, \varphi_{x}, \varphi_{y}$, is given by the canonical equations of the Hamiltonian $2 \mathrm{H}_{s 1}$, where $H_{s 1}$ is the slowly varying Hamiltonian Eq. (3), which is time dependent, and a procedure to remove the time dependency is desirable in order to identify invariants of motion.

Differently from previous works, we find that there is an infinite set of canonical transformations of the form

$$
\begin{aligned}
& \tilde{a}_{x}=a_{x}, \\
& \tilde{\varphi}_{x}=\varphi_{x}+t_{x} 2 \pi \Delta_{r} s / L, \\
& \tilde{a}_{y}=a_{y}, \\
& \tilde{\varphi}_{y}=\varphi_{y}+t_{y} 2 \pi \Delta_{r} s / L ;
\end{aligned}
$$


all suitable for creating a system of canonical coordinates $\tilde{a}_{x}, \tilde{\varphi}_{x}, \tilde{a}_{y}, \tilde{\varphi}_{y}$, where the Hamiltonian Eq. (3) becomes time independent. The coefficients $t_{x}, t_{y}$ shall here satisfy the condition $t_{x}+2 t_{y}=1$. Therefore, by assigning a value to $t_{x}=\tau$ we completely fix one possible system of canonical coordinates.

In the system $\tau$, the new coordinates are subjected to the dynamics of a time-independent Hamiltonian via the canonical equations. The simplest solution of the canonical equations $\tilde{a}_{x}^{\prime}=\tilde{\varphi}_{x}^{\prime}=\tilde{a}_{y}^{\prime}=\tilde{\varphi}_{y}^{\prime}=0$ is found to be

$$
\begin{aligned}
\tilde{a}_{y} & =\frac{\left(2 \pi \Delta_{r}\right)^{2}}{4 \Lambda^{2} L^{2}} \tau(1-\tau), \\
\tilde{a}_{x} & =\frac{\left(2 \pi \Delta_{r}\right)^{2}}{16 \Lambda^{2} L^{2}}(1-\tau)^{2}, \\
\tilde{\varphi}_{x}+2 \tilde{\varphi}_{y}+\alpha & =\pi M,
\end{aligned}
$$

with $M$ an integer of either 0 or 1 . The values of $\tau$ are limited to $0 \leq \tau \leq 1$.

Any one of these solutions $\tilde{a}_{x}, \tilde{\varphi}_{x}, \tilde{a}_{y}, \tilde{\varphi}_{y}$ can be transformed to the laboratory frame via Eqs. (4) and (2) at a specific longitudinal position along the accelerator. To simplify the discussion, but without losing generality, we take the $x-y$ projection of the Poincare surface of section and find that the particle coordinates lie on the curve

$$
\begin{aligned}
& x(t)=\sqrt{\beta_{x} a_{x}} \cos (-2 t-\alpha+\pi M), \\
& y(t)=\sqrt{\beta_{y} a_{y}} \cos (t),
\end{aligned}
$$

where $t$ is a variable that parametrizes the curve. We find that after each accelerator turn an initial point $\left(x, x^{\prime}, y, y^{\prime}\right)_{0}$, identified by $\tilde{a}_{x}, \tilde{a}_{y}, \tilde{\varphi}_{x}, \tilde{\varphi}_{y}$ and satisfying Eq. (5), is mapped to another point $\left(x, x^{\prime}, y, y^{\prime}\right)_{1}$, and all such points belong to a closed one-dimensional curve, which in the $x-y$ projection has the analytic form of Eq. (6). At the same time $\left(x, x^{\prime}, y, y^{\prime}\right)_{1}$ in the system $\tau$ keep unchanged the value of the variables $\tilde{a}_{x}, \tilde{a}_{y}, \tilde{\varphi}_{x}, \tilde{\varphi}_{y}$. Similar results can be found in any projection of the Poincaré surface of section.

We call this curve a 2D "fixed line" [15] based on its property that the particle coordinates will remain on this curve indefinitely. In analogy to 1D fixed points, we find that 2D fixed lines can be either stable or unstable. Figure 1 shows an example of several projections of a Poincare surface of section of a stable 2D fixed line. For convenience we show the classical horizontal and vertical projections $x-x^{\prime}, y-y^{\prime}$ in Figs. 1(a) and 1(b), which describe circles guaranteeing that $\tilde{a}_{x}, \tilde{a}_{y}$ are constant. Figure 1(c) shows the $x-y$ projection and $1(\mathrm{~d})$ shows the $x^{\prime}-y^{\prime}$ projection.

We have therefore reached the situation where in each system $\tau$ there is one stationary point that in the laboratory frame becomes a distinct fixed line. Therefore, we can conclude that the $4 \mathrm{D}$ phase space is populated by an infinite set of fixed lines dependent on the parameter $\tau$. These lines (a)

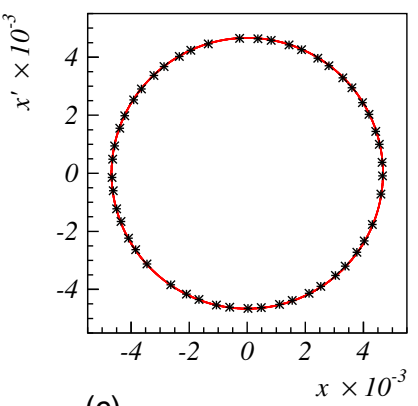

(c)

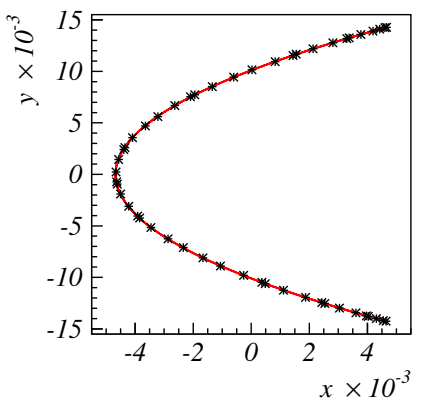

(b)

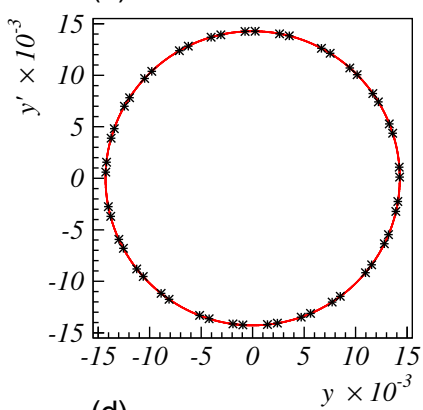

(d)

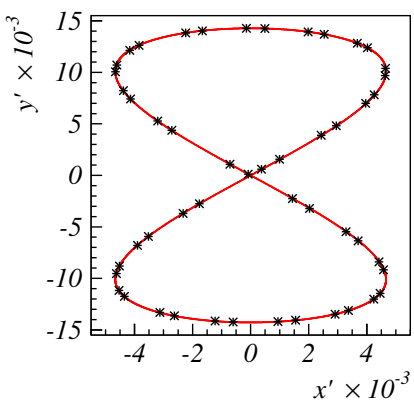

FIG. 1 (color online). The 2D fixed line as predicted by the theory (red dots) and position of a particle from simulations (black markers). The circular shape in (a),(b) shows that $\tilde{a}_{x}, \tilde{a}_{y}$ are constants of motion. In (c),(d) is clearly visible the unusual pattern deriving from the coupling created by the resonance.

are all close to one another so as to form a complex structure in the 4D phase space.

It is an interesting feature of the canonical equations in the system $\tau$ that they lead to the invariant of motion $2 \tilde{a}_{x}-\tilde{a}_{y}=C$. If we consider the same particle in another system $\tau^{\prime}$, the value of $C, \tilde{a}_{x}$, and $\tilde{a}_{y}$ remain the same. This is due to the fact that the parameter $\tau$ does not play a physical role, while the variables $\tilde{a}_{x}, \tilde{a}_{y}$ have a physical meaning of particle emittance (or action). This is not the case for the variables $\tilde{\varphi}_{x}, \tilde{\varphi}_{y}$, which depend on $\tau$. As the physics should not be dependent on $\tau$, we combine $\tilde{\varphi}_{x}, \tilde{\varphi}_{y}$ to eliminate $t_{x}, t_{y}$ by taking $\Omega=\tilde{\varphi}_{x}+2 \tilde{\varphi}_{y}: \Omega$ has the same value in any system $\tau$ [see Eq. (4)]. This means that the variables $\tilde{a}_{x}, \Omega$ are more convenient to characterize the dynamics as they are independent of $\tau$. Reexpressing the time independent Hamiltonian of any system $\tau$ into these variables we find a function neither dependent on time nor on $\tau$, i.e., an invariant of motion $I\left(a_{x}, \Omega\right)$. In the laboratory frame we can recast it in conveniently "scaled" coordinates as

$$
\hat{I}\left(\hat{a}_{x}, \Omega\right)=\mu \sqrt{\hat{a}_{x}}\left(\hat{a}_{x}-\xi\right) \cos (\Omega+\alpha)+\hat{a}_{x},
$$

with $\hat{a}_{x}=\left[4 L \Lambda /\left(2 \pi \Delta_{r}\right)\right]^{2} a_{x}, \hat{a}_{y}=\left[4 L \Lambda /\left(2 \pi \Delta_{r}\right)\right]^{2} a_{y}, \xi=$ $\left[4 L \Lambda /\left(2 \pi \Delta_{r}\right)\right]^{2} C / 2$, and $\mu=\Delta_{r} /\left|\Delta_{r}\right|$. Given the initial coordinates of a particle, we fix $\hat{I}, \xi$; hence, the associated level line of Eq. (7) yields the particle trajectory in $\hat{a}_{x}, \Omega$ coordinates. This allows us to predict the stability of all 
(a)

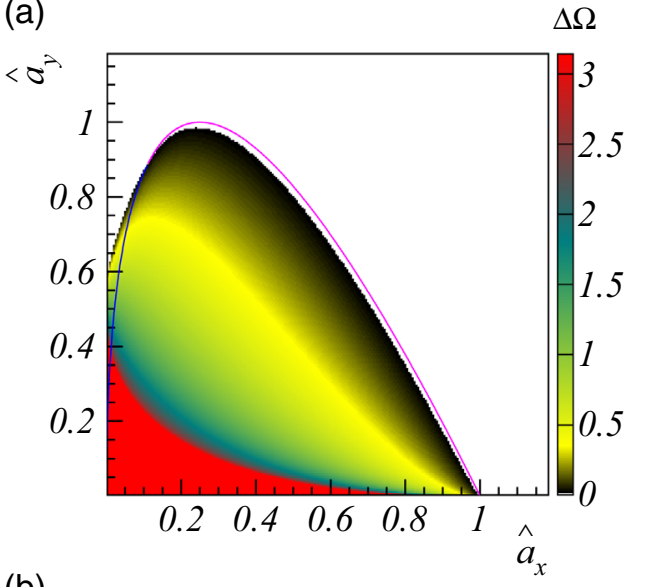

(b)

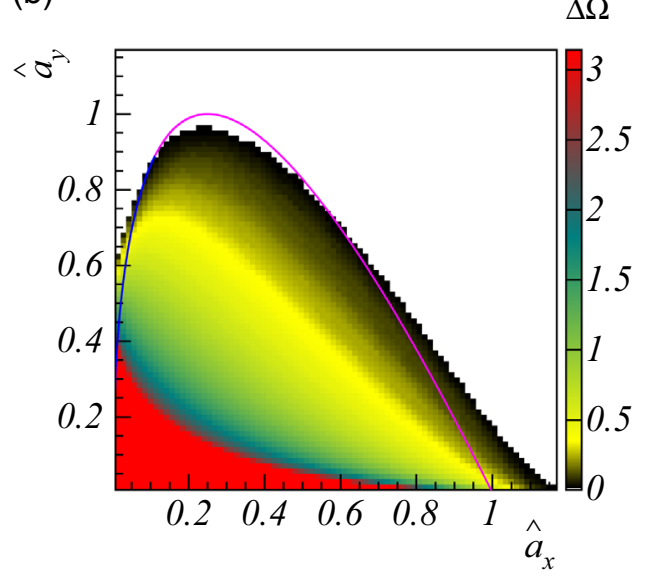

FIG. 2 (color online). Complete stability domain. Part a): the stability domain as obtained by the analytic theory for the GSI heavy-ion synchrotron SIS18 lattice with a distribution of sextupolar errors. Part b) shows the stability domain of SIS18 obtained with simulations for one single sextupolar kick.

particles according to the value of the two invariants $\hat{I}, \xi$. An analysis of the stability of motion using Eq. (7) reveals that the edge of stability of particles is consistent with the position of the fixed lines. This is shown in part (a) of Fig. 2 where the set of the fixed lines described by Eq. (5) forms a paraboliclike curve (blue and pink) in the $\left(\hat{a}_{x}, \hat{a}_{y}\right)$ plane. If a particle has initial values $\hat{a}_{x}, \hat{a}_{y}$ "inside" the set of the fixed lines, its stability depends on the value of $\Omega$. The pink line is the collection of $\hat{a}_{x}, \hat{a}_{y}$ with unstable fixed lines, and the blue line is the collection of the values $\hat{a}_{x}, \hat{a}_{y}$ with stable fixed lines. The color code in the pictures provides the set of allowed $\Omega$ as a function of $\hat{a}_{x}, \hat{a}_{y}$. This range is determined by the level line associated with the invariant $\hat{I}$ of a fixed line according to the following procedure: given a particle with certain $\hat{a}_{x}, \hat{a}_{y}$ values we find $\xi=\hat{a}_{x}-\hat{a}_{y} / 2$, which identifies a line that always intercept the pink line collection of the unstable fixed lines. To that interception point corresponds one value of $\tau$, and one value $\hat{I}_{f l}$ of the invariant Eq. (7). The angles $\Omega$ for which the motion of a particle with initial $a_{x}, a_{y}$ is stable are found in an interval of size $2 \Delta \Omega$, with

$$
\Delta \Omega\left(\hat{a}_{x}, \hat{a}_{y}\right)=\operatorname{arcos}\left[\frac{1}{\mu} \frac{\hat{I}_{f l}-\hat{a}_{x}}{\sqrt{\hat{a}_{x}}\left(\hat{a}_{x}-\xi\right)}\right],
$$

where we take $0 \leq \Delta \Omega \leq \pi$. Part (a) of Fig. 2 shows a red wide region where $\Delta \Omega=\pi$; there $\Omega$ can assume any value and the particle remains stable, other regions of the picture exhibit partial stability. Part (b) of Fig. 2 shows a comparison with computer simulation for the case with one single sextupolar error. The widening of the stability domain shown in Fig. 2(b) is due to the effect of higher harmonics, which are all excited by a single localized error. Further simulations show that distributed errors generated to mainly excite a single harmonic component yield a better agreement between theory and simulations.

Figure 2 shows another interesting feature: particles close to the $\hat{a}_{y}$ axis can be stable even "outside" the border of the stable 2D fixed lines. This demonstrates the existence of stable 2D "tori" as analogous to the stable "islands" in 1D dynamics. Contrary to the 1D case, these stable tori exist even without stabilizing detuning terms from higherorder multipolar components.

In conclusion, in proximity to the coupled $2 \mathrm{D}$ resonance $Q_{x}+2 Q_{y}=N$ there are 2D fixed lines that are equivalent to fixed points in one dimension. These lines are onedimensional closed curves in the 4D phase space. Their number is infinite, and they may either be stable or unstable. Our analysis is focused on distributions of sextupolar errors around a circular machine and should be seen as a general extension of previous studies [16,17]. Our study links the stability domain to the existence of fixed lines. We also find that the properties of stability define a scaling law, naturally associated with the scaling coefficient $\left[4 L \Lambda /\left(2 \pi \Delta_{r}\right)\right]^{2}$. The general properties are summarized in Fig. 2 expressed in scaled emittances. The full derivation of this theory will be part of a future publication [13].

This study constitutes a milestone for the studies with space charge and the process of 2D fixed line crossing and trapping phenomena in two dimensions induced by space charge, and in conjunction with synchrotron oscillation.

The research leading to these results has received funding from the European Commission under the FP7 Research Infrastructures Project EuCARD-2, Grant Agreement No. 312453.

[1] B. V. Chirikov, Phys. Rep. 52, 263 (1979).

[2] A. J. Lichtenberg and M. A. Lieberman, Regular and Chaotic Dynamics, 2nd ed. (Springer-Verlag, New York, 1992).

[3] R. Hagedorn, CERN Report No. CERN 57-1, 1957; R. Hagedorn and A. Schoch, CERN Report No. CERN 57-14, 1957.

[4] A. Schoch, CERN Report No. CERN 57-23, 1958; G. Guignard, CERN Report No. CERN 78-11, 1978. 
[5] E. D. Courant and H. S. Snyder, Ann. Phys. (Paris) 3, 1 (1958).

[6] H. Poincaré, Les Méthodes Nouvelles de la Mécanique Céleste, Vols. 1-3 (Gauthier Villars, Paris, 1899).

[7] W. Hardt, Report No. PS-DL-LEAR-Note-81-6, 1981; R. Cappi and M. Giovannozzi, Phys. Rev. ST Accel. Beams 7, 024001 (2004).

[8] P. Spiller and G. Franchetti, Nucl. Instrum. Methods Phys. Res., Sect. A 561, 305 (2006).

[9] J. Coupard et al., LIU Technical Design Report (TDR), CERN-ACC-2014-0337.

[10] G. Franchetti et al., Phys. Rev. ST Accel. Beams 13, 114203 (2010).

[11] G. Franchetti, I. Hofmann, M. Giovannozzi, M. Martini, and E. Metral, Phys. Rev. ST Accel. Beams 6, 124201 (2003);
E. Metral, G. Franchetti, M. Giovannozzi, I. Hofmann, M. Martini, and R. Steerenberg, Nucl. Instrum. Methods Phys. Res., Sect. A 561, 257 (2006).

[12] E. Forest, Beam Dynamics A New Attitude and Framework (Harwood Academic Publishers, Chur, Switzerland, 1998).

[13] G. Franchetti and F. Schmidt, Phys. Rev. ST Accel. Beams (to be published).

[14] A. W. Chao and M. Month, Nucl. Instrum. Methods 121, 129 (1974).

[15] F. Schmidt, DESY HERA Report No. 88-02, 1988 (unpublished).

[16] E. Todesco, Phys. Rev. E 50, R4298 (1994).; M. Gemmi and E. Todesco, Celest. Mech. Dyn. Astron. 67, 181 (1997).

[17] M. N. Vrahatis, H. Isliker, and T.C. Bountis, Int. J. Bifurcation Chaos Appl. Sci. Eng. 07, 2707 (1997). 